\title{
La Perception De L'image De Marque Automobile: Étude Qualitative Exploratoire Auprès Des Consommateurs De La Ville d'Agadir
}

\author{
Mohammed Hicham Hamri (P.E.S) \\ Amira Qanqom (PhD Student)
}

ERETTLOG Laboratory, National School of Commerce and Management of Agadir, ENCG-Agadir, University Ibn Zohr, Morocco

Doi:10.19044/esj.2018.v14n25p76 URL:http://dx.doi.org/10.19044/esj.2018.v14n25p76

\begin{abstract}
This paper focuses on conducting an exploratory study that allows us to discover how people in Agadir city can construct an image of an automobile brand. Indeed, in the automotive sector, brands are very important and the image is one of the priorities to last on the market. As a result, a lot of companies have attached a significant importance to the measurement of their brand image to consumers. In this study, we are going to base our work on the main typologies of brand image, namely Korchia (2001), which allows its measurement. Also, we will take a case study of them in regards to our field of study. At the end of this paper, we aim to design our own typology. For that purpose, we conducted a qualitative study, by individual interviews. As a conclusion, this work is dedicated to develop a brand image typology, specific to automotive sector, and adapted to Agadir city consumers.
\end{abstract}

Keywords: Brand image, automobile, typology, perception

\section{Résumé}

Cet article a pour but de mener une étude exploratoire nous permettant de soulever les moyens de perception de l'image de marque automobile dans le grand Agadir. En effet, dans le secteur automobile, les marques sont les reines et l'image représente l'une des priorités pour perdurer sur le marché. Pour cette raison, les entreprises accordent une grande importance à la mesure de leur image de marque auprès des consommateurs. À ce titre, en se basant sur les principales typologies de l'image de marque qui sont indispensables pour sa mesure, ce papier se voue à confronter l'une d'entre elles, à savoir celle de Korchia (2001), à notre terrain d'étude, à l'issue de laquelle fut conçue notre propre typologie. Pour ce faire, nous avons mené une étude qualitative moyennant des entretiens individuels, étude à travers laquelle nous visons à 
développer une typologie de l'image de marque automobile adaptée aux consommateurs du grand Agadir.

Mots-clés : Image de marque, automobile, typologie, perception

\section{Introduction}

Selon Aaker (2011), l'image de marque représente la concrétisation de la stratégie de positionnement. Elle englobe la somme des perceptions et des émotions reliées à la marque. Grâce aux travaux de Heude (1990), il a été prouvé que cette dernière est perçue par le consommateur à travers des signes permettant de connaître ou reconnaître une certaine marque, et ainsi s'en créer une image. Ces signes peuvent provenir de divers horizons, mais ils véhiculent automatiquement l'image de marque. Il existe par exemple la publicité, le bouche à oreille, le nom de la marque, le logotype et plusieurs autres éléments.

Afin d'étudier la perception de l'image de marque, il est impératif de disposer d'une typologie. Pour avoir une meilleure connaissance de principales typologies créées avant, nous allons commencer par synthétiser celles-ci. Suite à cela, nous nous baserons sur l'une d'elles afin de conduire notre étude exploratoire, ayant pour but de définir notre propre typologie de l'image de marque. Cette dernière sera spécifique à notre terrain d'étude.

\section{1- Les principales typologies de l'image de marque}

Keller (1993), Aaker (1994) et Korchia (2001), ont chacun établi une typologie distincte, basée principalement sur les associations à la marque ; en d'autre termes, ce sont des nœuds liés à la marque (Korchia, 2001). Les associations à la marque peuvent être aussi définies comme les nœuds informationnels liés au nœud de la marque dans la mémoire, lesquels contiennent le sens de la marque pour le consommateur (Keller, 1993).

Figure 1. Typologie de l'image de marque selon Keller

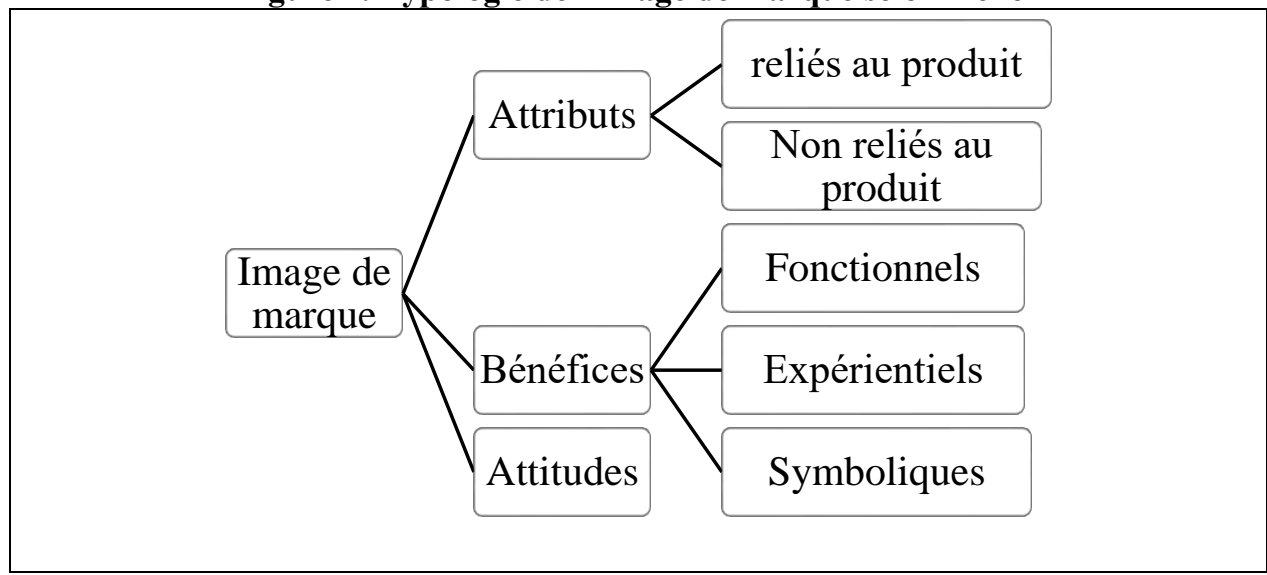

Source : Keller (1993) 
Figure 2. Les dimensions de l'image de marque

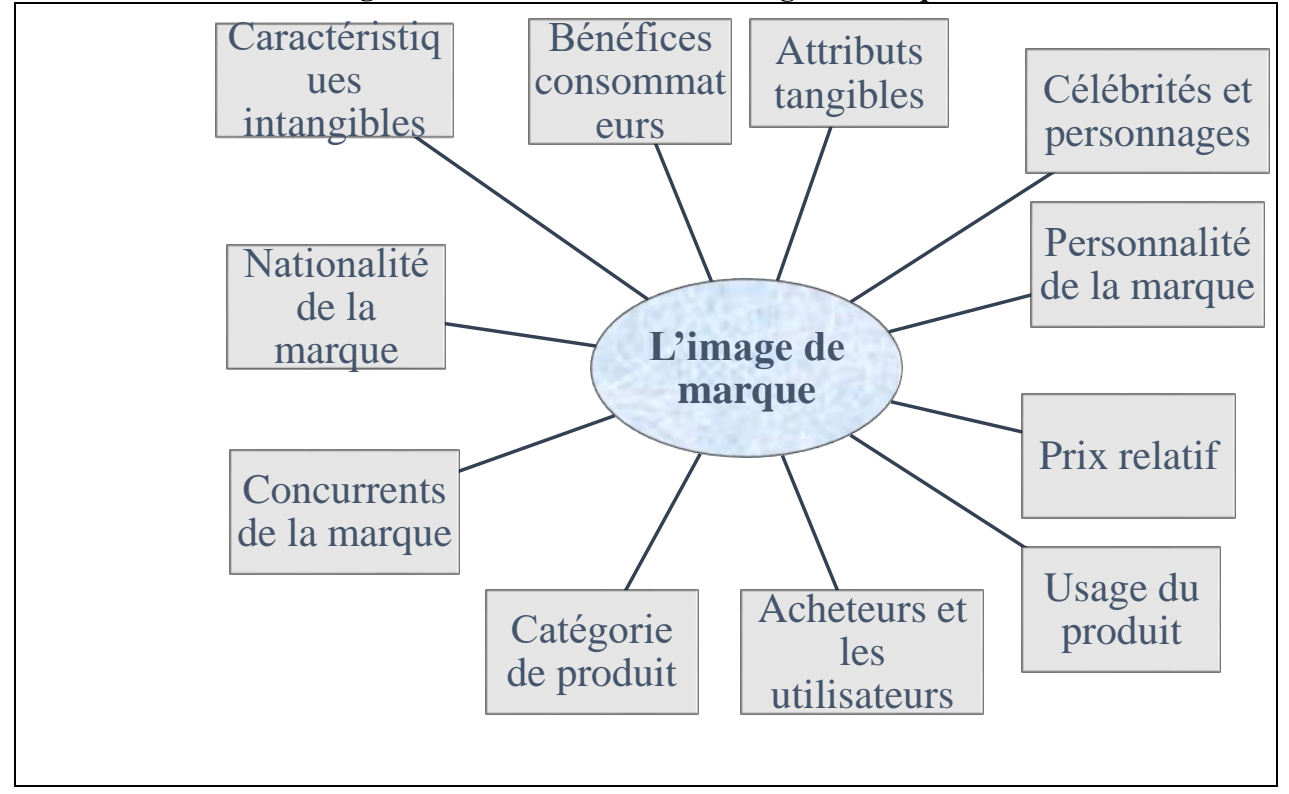

Source : Aaker (1994)

Figure 3. Typologie de l'image de marque selon Korchia

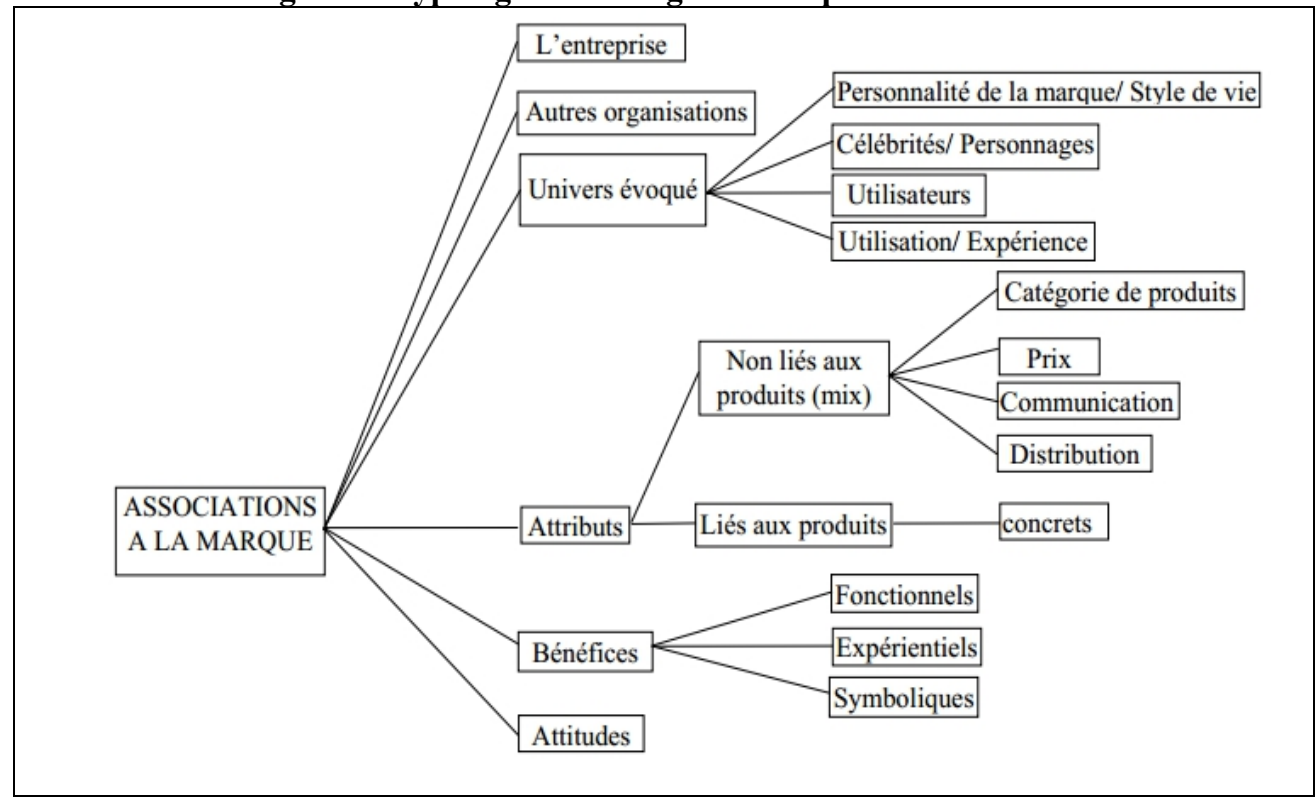

Source : Korchia (2001)

Les typologies de Keller (1993) et d'Aaker (1994) présentent plusieurs points de divergence, mais elles possèdent également des similitudes. In fine, aucunes de ces typologies n'est complète et selon Korchia (2001), elles ne sont pas exhaustives. Dans le cadre de notre recherche, nous estimons que la 
typologie de Korchia est plus complète, et comporte des catégories assez variées et diverses.

Notre recherche a pour but de dégager les supports de perception de l'image de marque automobile d'un consommateur. Les associations à la marque ont un rôle primordial, dès lors les typologies élicitées auparavant sont la base de notre travail et constituent les éléments clés qui vont nous permettre de mener notre recherche. Notre choix s'est porté sur la typologie de Korchia (2001), qui est la plus récente, et qui a remis en cause les précédentes typologies.

Evrard et al. (2000) précisent que dans le cadre des champs peu explorés et pour lesquels aucune théorie satisfaisante n'a pu être préalablement étayée, la conduite d'une étude qualitative de nature exploratoire semble une démarche privilégiée. Le recours à une méthodologie qualitative est avant tout le résultat d'un choix raisonné. La méthode qualitative, est adaptée dans les approches exploratoires car elle permet une collecte de données plus rapide et plus centrée, même si elle porte sur un nombre assez restreint d'interviewés.

\section{2- Approche qualitative et Choix de la méthode des Entretiens individuels}

Il existe plusieurs méthodes de collecte de données. Dans notre cas, nous voulons obtenir des informations ciblées, relatives aux perceptions des consommateurs. En effet, pour pouvoir mesurer de manière exhaustive la perception de l'image de marque par le consommateur, nous devons étudier les associations à la marque détenues dans la mémoire du consommateur, en s'assurant que celles-ci peuvent être identifiées de manière complète. Cela ne peut être réalisé que si l'on détient une typologie efficace ; dans le cas inverse, la perception des consommateurs serait mesurée de façon inefficace.

Dans la méthodologie qualitative, différentes méthodes et techniques peuvent être utilisées, dont les plus répandues sont les entretiens et la méthode des cas. Cependant, nous savons déjà que les chercheurs dans le domaine de l'image de marque (Korchia, 2001; Michel, 2009), préconisent l'usage des entretiens, et spécialement les entretiens individuels, au détriment des entretiens de groupe. A cet égard, nous optons pour la méthode des entretiens individuels, afin de conduire notre étude qualitative.

Les entretiens relèvent des méthodes qualitatives. Trois types d'entretien individuels sont distingués : non directif, semi directif ou directifs, et les tests projectifs (individuels ou de groupe). Baumard et Ibert (2007) rappellent que l'entretien est une technique utilisée pour collecter des données discursives résultant des interviews. Les entretiens individuels non directifs sont peu utilisés en sciences de gestion car ils permettent de recueillir un discours qu'aucune logique extérieure ne vient à priori structurer par des questions directes ou standardisées. Toutefois, nous pouvons affirmer que les chercheurs 
recourent beaucoup plus aux entretiens semi-directifs, conduits habituellement par un guide d'entretien comprenant les thèmes ou les axes de la recherche.

Cet outil est probablement le mode de recueil de données qualitatives le plus utilisé en recherche comme en études marketing (Evrad et al., 2000). Nous avons enregistré vocalement chaque interview, pour pouvoir le retranscrire ultérieurement.

Le temps qui nous sera accordé par chaque personne sera précieux et ne nous permettra surement pas un entretien non directif car celui-ci demanderait plus de temps. Il nous faudra donc cibler les éléments de réponse, qui doivent être pertinents pour répondre à notre objectif. En ce qui concerne la méthode de collecte, nous avons choisi de procéder par des entretiens semi-directifs. Ceux-ci seront menés à l'aide d'un guide d'entretien que nous avons élaboré en nous basant sur la typologie de Korchia (2001).

\section{- Le guide d'entretien}

Nos entretiens sont basés sur le mode de conversation, et nous avons tenu à adapter l'ordre et la teneur des questions selon les réponses de nos sujets (Demers, 2003). De plus, nous avons veillé à personnaliser avec un maximum d'exhaustivité notre guide d'entretien, suivant les consignes de LoufraniFedida (2006), qui stipule que «le guide d'entretien n'est pas un questionnaire ouvert mais un aide-mémoire qui permet de vérifier qu'aucun point important n'a été oublié ».

La conception de notre guide d'entretien s'est également basée sur ce qu'on appelle la procédure d'élicitation libre. Celle-ci est fondée sur le principe de diffusion de l'activation. C'est le processus qui se déroule lors de l'accès à une information stockée en mémoire (Anderson, 1983 ; Keller, 1993). Lorsque le niveau d'activation à un nœud (par exemple une association à une marque), est supérieur à un certain seuil, l'information contenue dans ce nœud est rappelé en mémoire et elle devient de ce fait disponible. Par conséquent, les nœuds les plus fortement liés à ce dernier vont également être rappelés.

Un stimulus de départ, qui peut être représenté en une courte phrase, a pour but d'activer un nœud informationnel. Par exemple, si on prend un nom de marque comme stimulus, notre objectif est d'activer tous les nœuds relatifs à ce nom dans l'esprit du consommateur. Cependant, le problème se pose lorsqu'un consommateur ne peut pas se remémorer tout ce qu'il stock dans sa mémoire concernant une marque, en réponse à une seule question, ou à un seul stimulus. De ce fait, Brucks (1985) affirme qu'on peut avoir recours à une procédure d'élicitation multiple, qui stipule que chaque concept élicité peut servir de stimulus par la suite. Cette procédure peut durer aussi longtemps que le consommateur interviewé est capable de verbaliser de nouveaux concepts.

Compte tenu du fait que notre échantillon est composé de personnes disposant d'une voiture, et également de celles désirant en acheter, nous avons 
adopté notre discours en fonction de ce critère. Par conséquent, nous demandons aux personnes motorisés des informations concernant leur propre marque de voiture, et questionnons les autres sur la marque de voiture qu'ils désirent acquérir. En effet, il est important de ne pas négliger les acheteurs potentiels de voiture, car contrairement à ce que l'on pourrait croire, ils ont une image bien construite des marques automobiles.

Avant de commencer les interviews, nous avons présenté notre travail et l'objectif que nous avons à travers cette étude, ceci dans le but de les guider quant à la nature des informations que nous désirons obtenir d'eux. Ensuite, nous les laissions seuls 10 à 15 minutes afin de se préparer mentalement, et organiser leur propos. À la fin de chaque interview, nous leur fournissions un tableau contenant les éléments constituant la typologie de l'image de marque qu'ils devaient classer par ordre d'importance par rapport à leur perception de l'image de marque. Ces catégories sont présentées de façon très discrète, c'està-dire que nous y faisions allusion sans les éliciter proprement. Enfin, les entretiens ont eut une durée allant de 35 à 70 minutes.

Les résultats obtenus seront recensés à l'aide de la typologie choisie, en l'occurrence celle de Korchia, soit pour la confirmer ou éventuellement donner lieu à une nouvelle typologie adaptée à notre contexte de recherche.

\section{- Choix des sujets (interviewés)}

En nous basant sur les préconisations de (Thiétart, 2017) «la taille adéquate d'un échantillon est celle qui permet d'atteindre la saturation théorique. Cette saturation théorique est atteinte lorsqu'on ne trouve plus d'information supplémentaire capable d'enrichir la théorie...).

De ce fait, nous nous sommes arrêtés à 23 personnes, dix femmes et treize hommes, du fait qu'à partir du vingtième questionnaire, les informations commençaient à se répéter. Ils étaient âgés d' entre 23 et 58 ans, ayant tous leur permis de conduire, et possédant ou désirant posséder une voiture. Ce sont toutes des personnes actives, de différentes catégories socioprofessionnelles (cadres, directeurs, secrétaires, commerciaux...), et aussi de classes sociales différentes. Toutes ces personnes ont un intérêt pour l'automobile, connaissent les différentes marques qui existent sur le marché, et sont donc des consommateurs ou futurs consommateurs.

\section{- Résultats obtenus}

Avant de présenter les résultats, il convient d'expliquer comment nous avons recensé les propos de chaque sujet.

Pour analyser les verbatims, nous avons suivi les préconisations de Miles et Huberman (2003) qui consistent en trois étapes. Pour la première étape, nous nous sommes basés sur les catégories définies dans la typologie de Korchia (2001).

La deuxième phase de mise en forme a facilité l'organisation des données collectées afin d'émettre des propositions finales. Nous avons ensuite cherché 
à définir les conceptions des interviewés de leurs perceptions d'image de marque (analyse inter-cas). La dernière étape a permis de tirer et de vérifier les conclusions émises lors de la deuxième phase.

Comme cité auparavant, la typologie de Korchia (2001), représente la base de la conception du guide d'entretien, et également de son analyse. Nous avons adapté les catégories qui la constituent de la façon suivante :

- L'entreprise : contient le pays d'origine, la stratégie, mais également les propos qui portent sur la notoriété de la marque ;

- Autres organisations : concurrents, gouvernement...;

- Personnalité de la marque : traits de la personnalité humaine associée à la marque, des propos comme belle, élégante... peuvent y figurer ;

- Célébrités, événements: par exemple le célèbre pilote automobile « Mehdi Bennani », associé à la marque Honda, et le « Grand Prix de Marrakech de Formule 1 », qui est un événement qu'on peut également associer à cette même marque ;

- Utilisateurs : Association entre la marque et le type d'utilisateurs de celle-ci, qui peuvent être établis à partie de leur âge, classe-sociale, physique....;

- Usage, expérience : L'utilisation personnel du produit ; cette catégorie inclut si la personne évoque son expérience personnelle avec la marque par exemple ;

- Catégorie de produits : les catégories de produits que couvre la marque, par exemple les différents modèles de voitures, les motos, les quads, les zodiacs...;

- Prix : on associe souvent le prix à la qualité du produit ou service ;

- Communication : tout ce qui est lié à la communication de l'entreprise (publicité, actions promotionnelles...), en dehors les célébrités et événements car ils figurent dans une catégorie à part entière ;

- Distribution : ici on cible particulièrement les points de vente, en l'occurrence les concessionnaires de la marque, et tout ce qui s'y relie (SAV, commerciaux, personnel...);

- Attributs liés au produit : tout ce qui est lié à la composition physique du produit de la marque (carrosserie, pièces, moteur, climatisation, pneus, options...).Nous avons inclus ici les adjectifs liés aux attributs du produit, par exemple (robuste, performante, bonne qualité...) ;

- Bénéfices fonctionnels : on parle ici principalement des besoins de sécurité qu'une voiture peut combler, ou éliminer le problème de transport ;

- Bénéfices expérientiels : le plaisir que l'utilisation du produit procure ;

- Bénéfices symboliques : le besoin d'estime et de différenciation; on parle ici de tout ce qui est lié aux besoins d'expression personnelle ; 
- Attitude : sentiment affectif envers la marque, des propos comme (j'aime la marque, c'est ma marque préférée...) peuvent y figurer ;

- Propos non codés : tout ce qui n'entre dans aucune des catégories précitées.

Nous avons obtenu au total 995 propos. Le nombre de propos élicités par les interviewés varient entre un minimum de 23 fois et 56 fois par entretien. Certains propos ont été répété deux ou plusieurs fois par un même sujet ; ceuxci n'ont été comptés qu'une seule fois. Seulement 8 propos n'ont pu être codés du fait que nous n'avons pu l'intégrer dans aucune des catégories.

Nous avons utilisé le logiciel Excel pour saisir l'ensemble des codes correspondants à chaque catégorie, et ce pour chaque entretien.

Tableau 1. Nombre d'associations à la marque (AM) élicitées par catégorie

\begin{tabular}{|c|r|r|r|}
\hline Catégorie & $\begin{array}{c}\text { Nb d'associations à } \\
\text { la marque }\end{array}$ & Pourcentage & Classement \\
\hline Prix & 152 & $15,28 \%$ & 1 \\
\hline Attributs liés aux produits & 144 & $14,47 \%$ & 2 \\
\hline Facteurs intrinsèques & 136 & $13,67 \%$ & 3 \\
\hline Attitude & 124 & $12,46 \%$ & 4 \\
\hline Distribution & 97 & $9,75 \%$ & 5 \\
\hline Personnalité de la marque & 84 & $8,44 \%$ & 6 \\
\hline Catégorie des produits & 79 & $7,94 \%$ & 7 \\
\hline Communication & 60 & $6,03 \%$ & 8 \\
\hline Utilisateurs & 35 & $3,52 \%$ & 9 \\
\hline Autres organisations & 30 & $3,02 \%$ & 10 \\
\hline Bénéfices fonctionnels & 15 & $1,51 \%$ & 11 \\
\hline Bénéfices symboliques & 12 & $1,21 \%$ & 12 \\
\hline Usage, expériences & 10 & $1,01 \%$ & 13 \\
\hline Propos non codés & 8 & $0,80 \%$ & 14 \\
\hline Bénéfices expérientiels & 5 & $0,50 \%$ & 15 \\
\hline Célébrités, événements & 4 & $0,40 \%$ & 16 \\
\hline Totale des AM élicitées & 995 & $100,00 \%$ & \\
\hline
\end{tabular}

Source : Conception personnelle

La collecte des données lors des entretiens a rencontré plusieurs obstacles du fait que la majorité des interviewés éprouvaient des difficultés à s'exprimer librement alors que leurs propos étaient enregistrés par voie audio. La fréquence des associations dans les citations et les discours des interviewés étaient également réduits à cause du manque d'informations précises sur les marques. Ce fut le cas en particulier des femmes. Par contre, les hommes avaient une idée bien précise et structurée de la question. Le nombre de propos élicités non codés, est de 8 , ce qui montre que la typologie est assez exhaustive et peut parfaitement être appliqué au secteur automobile, avec quelques 
ajustements. Selon Korchia (2001) «Les 15 catégories présentées peuvent être réduites en un nombre plus restreint de dimensions, qui peuvent varier suivant les marques. » ce qui nous autorise à adapter ces différentes catégories, selon notre cas, bien sûr en nous basant sur les résultats de notre étude qualitative.

Par conséquent, nous nous permettons de proposer une nouvelle typologie de l'image de marque qui sera parfaitement adaptée à notre champ d'étude et au contexte marocain.

\section{3- Conception d'une nouvelle typologie de l'image de marque}

Afin de concevoir une nouvelle typologie de l'image de marque, nous nous basons bien sûr, sur les résultats de l'étude qualitative, mais nous nous inspirons également de Ratier (2006), qui a élaboré un modèle illustrant les supports de perception de l'image de marque automobile en France. Ce dernier comprend les catégories suivantes :

- la publicité (publicité médias, publicité directe et publicité par l'événement)

- le prix

- les valeurs intrinsèques à la marque (passé de la marque, innovation, nom de marque, logo, côte à la revente, clientèle ciblée)

- les produits vendus (confort, étendue de la gamme, esthétique, fiabilité, qualité / finition, sécurité, image)

- les services annexes (qualité du réseau, SAV, personnel, services complémentaires)

- les facteurs externes (bouche-à-oreille, concurrence, presse, clientèle extérieure à la cible)

Figure 4. Modèle de perception de l'image de marque automobile selon (Ratier, 2006)

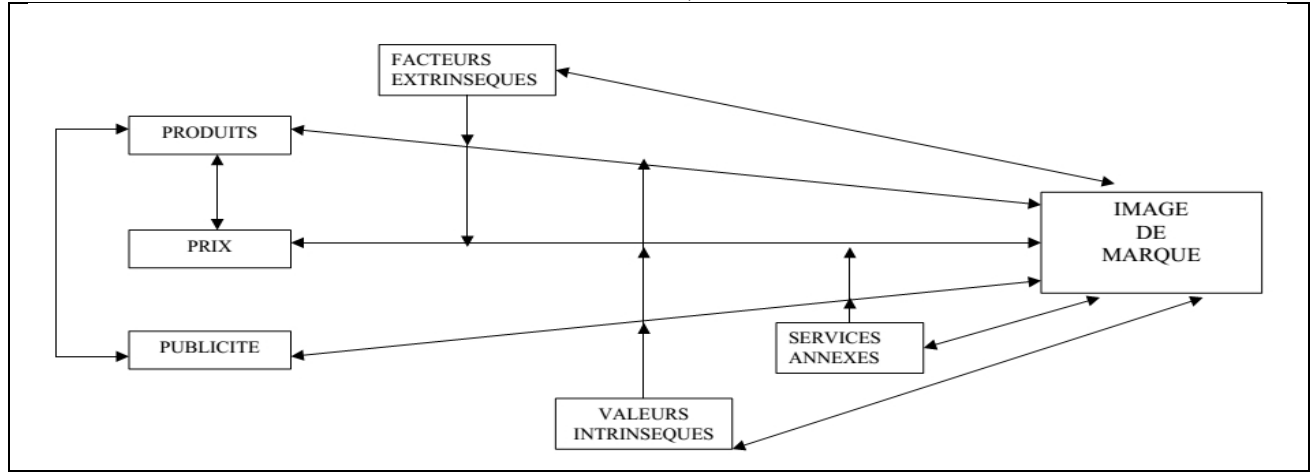

Source : Ratier (2006)

En effet, nous distinguons certaines des composantes de l'image de marque selon Korchia (2001) assemblées dans une même catégorie. Nous allons donc suivre cette logique, en nous appuyant sur les résultats de l'étude qualitative, en citant certains des propos des interviewés afin d'appuyer nos choix. 


\section{- Produit}

Les attributs liés aux produits sont les éléments les plus cités, et dans lesquels les personnes s'expriment le plus. Nous citons à titre d'exemple les propos suivant : " ... je dirai que c'est une voiture performante, robuste, ayant des options très attrayantes(...) je voyage souvent avec et je ne sens pas passer le temps, sa tenue de route est incomparable (...) elle est robuste, fiable et sécurisée(...) cependant je souffre souvent d'un manque d'espace dans les sièges arrières (...)». Nous avons remarqué que certaines catégories de la typologie de (Korchia, 2001) sont utilisées tout simplement en association au Produit, qui est dans notre cas «une voiture». Nous citons, en plus des attributs liés au produit :

- les catégories de produit : dans cette dimension de l'image de marque, les interviewés énumèrent les différents modèles de voiture qu'ils connaissent de la marque, et cela est évidemment enclenché quand on parle du produit.

- les bénéfices fonctionnels, expérientiels et symboliques : ces trois dimensions sont également étroitement liées au produit. A titre d'exemple, nous pouvons citer les propos de certains interviewés : «(...) je me sens en sécurité dans ma voiture (...) ce qui me fait le plus plaisir est que je ne prends plus de taxis (...) je fais la navette chaque jour, j'aimerais acheter une voiture pour ne plus me farcir tout ce trajet(...) ma fiancée voudrait que j'achète une voiture avant de demander sa main à son père... » Tous ces propos illustrent les avantages qu'une personne peut tirer de l'utilisation d'une voiture.

\section{- Le prix}

La totalité des interviewés ont mentionné "le prix » dans leur propos, souvent en l'associant à la qualité, aussi bien que les coûts associés à l'utilisation de la voiture et à son entretien. Il nous a parut donc logique d'intégrer ces éléments à cette catégorie, vu que les consommateurs y associent ces dépenses. A titre d'exemple nous citons : «(...) je trouve que c'est une voiture économique ; (...) son prix par rapport à sa qualité, je l'ai trouvé très correcte;(...) je fais le plein a presque 500dhs, et je roule avec pendant plus de 20 jours ;(...) je ne changerai pas cette voiture contre une autre pour laquelle je devrais payer une vignette et une assurance très chères ; (...) en général, en plus du prix de la voiture qui déjà n'est pas donné, il faut prévoir une petite fortune à coté : l'entretien, l'assurance, la vignette(...) »

\section{- La communication}

Dans cette catégorie les interviewés s'expriment majoritairement sur la publicité, le sponsoring, la presse spécialisée, les emails et les SMS. « (...) en vérité je reproche à Honda un manque de communication à Agadir, par contre sur Casa je remarque de temps en temps un peu de publicité mais jamais sur Agadir(...) J'ai beaucoup apprécié une publicité de Renault que j'ai vu sur TF1, je trouve que c'est très créatif (...) j'ai assisté une fois au festival dur rire d'Agadir qui été sponsorisé par Jaguar, et à l'entrée ils ont exposé certains 
modèles, je pense que ça a permis aux gens de découvrir plus la marque (...)j'ai découvert que Peugeot faisait une promotion très intéressante grâce à un email que j'avait reçu, (...) Seat m'envoi souvent des SMS avec les offres, c'est comme ça que j'ai informé mon frère qui voulait acheter une voiture de cette offre, (...) je trouve que les magazines spécialisées permettent au gens d'avoir une idée bien conçu de chaque voiture (...) »

\section{- La distribution}

Dans cette catégorie nous avons intégré des propos relatifs au point de vente, pour notre cas ce sont les concessionnaires et les revendeurs. Le mot distribution n'est donc pas adapté à notre contexte, ce qui nous pousse à le nommer «Point de vente ». A titre d'exemple nous citons : « (...) le concessionnaire m'a offert 5 vidanges gratuits (...) franchement je connais le directeur de la concession, c'est un gentil monsieur très serviable et professionnel (...) le concessionnaire d'Agadir m'a fait un mauvais coup, je ne ferai plus jamais affaire avec lui, j'ai du partir jusqu'à Casa pour m 'acheter la Mercedes avec les options que je veux (...) le? commercial est très compétant, $j$ 'avoue qu'il $m$ 'a fait aimé la marque (...) »

Les éléments qui précèdent, à savoir (Produit, Prix, Communication et Point de vente), nous amènent directement à penser aux composants du Mix Marketing, et c'est ainsi que nous allons donc nommer cette catégorie.

\section{- L'entreprise}

Selon la typologie adoptée, cet élément contient en général des propos relatifs à l'entreprise, la stratégie, la marque et le pays d'origine. Or, lors du déroulement des entretiens, les interviewés associaient ses éléments directement à la marque, en plus du nom, du logo, l'historique de la marque, en ajoutant l'implication écologique de cette dernière. De ce fait, en nous appuyant sur le modèle de (Ratier, 2006), qui a rassemblé les éléments cités dans une seule catégorie nommée « Valeurs intrinsèques à la marque », nous avons suivi cette même logique pour la retranscription des données, en ajoutant un nouvel élément qui nous est apparu grâce aux entretiens, qui est la valeur à la revente. A ce titre nous citons : «(...) J'ai toujours voulu posséder une Mercedes, pour moi c'est la meilleure (...) les voitures allemandes sont les plus robustes, et les françaises sont mal réputées (...) pour moi la France devrait laisser l'automobile aux allemands, c'est eux qui savent ce qu'ils font (...)Honda a beaucoup innové ces deux dernières années, je trouve les derniers modèles à la pointe de la technologie, si on compare avec les anciens (...)si je peux recommander quelque chose, je dirai que les marques automobiles devraient penser plus écologique (...) les marques devraient penser plus à concevoir des voitures hybrides, c'est la grande tendance et le Maroc devrait s'y lancer le plus vite (...) je l'ai choisi tout simplement parce que c'est une Audi, la marque parle d'elle-même (...)maintenant qu'elle a été rachetée par une entreprise allemande, les voitures de Seat sont plus robustes, et elle a une 
meilleure notoriété (...) Mercedes est très demandée sur le marché de l'occasion, je suis sûre que je n'aurai pas de mal à la revendre à bon prix "»

\section{- Les facteurs externes}

Selon Ratier (2006), cette catégorie comprend bouche-à-oreille, concurrence, presse, clientèle extérieure à la cible. Pour ce qui est de la typologie de Korchia (2001), il y figure l'élément «autres organisations » comprenant en particulier la comparaison avec la concurrence. Dans notre cas d'étude, et conformément aux résultats des entretiens, nous y intégrerons en plus des concurrents et du bouche à oreille, le profil des utilisateurs qui a beaucoup été mentionné. Nous citons : « (...) au départ je voulais acheter une Peugeot, mais un ami m'a dit qu'il regrette énormément d'en avoir acheté une, et franchement ça m'a fait réfléchir et j'ai changé d'avis (...) j'ai entendu dire qu'en route elle n'est pas très confortable (...) bien sûr que Volkswagen est meilleure que Renault, on compare l'incomparable là (...) si on compare Mercedes et Audi, je choisirai forcement Mercedes parce que c'est la meilleure (...) les voitures Honda, spécialement l'ACCORD est souvent achetée par les militaires, je remarque souvent des «accords 》 avec des immatriculations étatiques, ou conduites par une personne portant un uniforme militaire (...) c'est les riches qui roulent en Porsche (...) »

\section{- Attitude}

Rappelons que nous adoptons la conceptualisation affective de l'attitude, qui repose uniquement sur l'aspect émotionnel, et qui peut être positive ou négative. Par conséquent, cette catégorie contient des propos relatif à l'expression émotionnelle des interviewés par rapport aux marques automobiles. Nous citons « (...) ma marque préférée c'est Mercedes (...) j’ai une relation particulière avec la Honda, vraiment je ne peux pas expliquer pourquoi (...) Fiat me rappelle de vieux souvenirs, elle aura toujours une place dans mon cour (...) je déteste Skoda, je ne sais pas pourquoi mais je trouve que c'est une marque dévalorisante (...) ».

\section{- La personnalité de la marque}

Contrairement à nos attentes, les interviewés n'ont pas eu du mal à associer les marques de voiture à des traits de personnalité humaine ; plus que ça, certains décrivent spontanément leur marque de voiture en lui attribuant un adjectif relatif à la personnalité humaine. Nous citons les principaux traits que nous avons pu relever à travers les entretiens : "Performante, Technique, Fière, Amicale, Agréable, Sympathique, Généreuse, Frimeuse, Fiable, Classe, Créative, Branchée, Charmeuse, Forte, Moderne, Menteuse, Attachante, Joyeuse, Simple, Réservée »

La catégorie « usage et expérience » sera éliminée de la typologie retenue pour notre étude. En effet, les propos qui peuvent s'y référer entrent souvent dans d'autres catégories. En plus de cela, le modèle de Ratier ne contient pas cet élément, ce qui vient appuyer notre choix. En ce qui concerne les célébrités, 
il n'existe qu'un seul interviewé qui a cité « Mehdi Bennani », le célèbre pilote de Formule 1, en faisant référence à la marque Honda, ce qui nous a poussés à abandonner cet élément. De plus, les événements sont associés au sponsoring et parrainage, c'est pour cette raison qu'ils sont intégrés dans la catégorie Communication.

Enfin, les interviews nous ont permis de mettre la main sur un certain nombre d'items.

Le nombre de propos élicités et leur pourcentage montrent la composition de la perception de l'image de marque dans la mémoire du consommateur.

Dans le secteur que nous avons choisi d'étudier, une distinction doit être faite entre hommes et femmes, car le type mais aussi la quantité d'informations énoncées varient remarquablement. En effet, les hommes peuvent être qualifiés comme connaisseurs en la matière, ils sont très bien informés et s'égarent dans leur description du produit de la marque, contrairement aux femmes qui ont presque toutes le reflex de dire qu'elles ne connaissent pas grand-chose. Cependant, au cours de l'interview, il s'avère qu'elles ont une idée bien précise de la marque, mais un peu éloignée des détails techniques.

De ce qui précède, nous allons donc ajuster le tableau illustrant le nombre de propos obtenus grâce aux entretiens, de la manière qui suit (Tableau 2).

Tableau 2. Résultat de l'étude qualitative

\begin{tabular}{|c|c|c|c|}
\hline Catégories & $\begin{array}{c}\text { Nb d'associations à la } \\
\text { marque }\end{array}$ & Pourcentage & Classement \\
\hline Produit & 255 & $26,10 \%$ & 1 \\
\hline Prix & 152 & $15,56 \%$ & 2 \\
\hline $\begin{array}{c}\text { Valeurs intrinsèques à la } \\
\text { marque }\end{array}$ & 136 & $13,92 \%$ & 3 \\
\hline Attitude & 124 & $12,69 \%$ & 4 \\
\hline Point de vente & 97 & $9,93 \%$ & 5 \\
\hline Personnalité de la marque & 84 & $8,60 \%$ & 6 \\
\hline Facteurs externes & 65 & $6,65 \%$ & 7 \\
\hline Communication & 64 & $6,55 \%$ & 8 \\
\hline Totale & 977 & $100,00 \%$ & \\
\hline
\end{tabular}

Source : Conception personnelle

L'objectif de notre recherche est de mener une étude visant à déceler les supports de perception de l'image de marque dans l'esprit du consommateur, ce qui revient à détecter les associations à la marque. C'est pour cette raison qu'il est impératif de se baser sur une typologie de l'image de marque qui classe ces associations selon des catégories, et qui puisse englober tout type d'association. A défaut de cette typologie, certaines associations peuvent être négligées, et l'étude sera donc incomplète. Comme nous l'avons déjà mentionné, la typologie de Korchia (2001), est la base de 
notre nouvelle typologie, et en tenant en compte le modèle de Ratier (2006), nous arrivons à la typologie ci-dessous.

Figure 5.Typologie de l'image de marque retenue pour l'étude

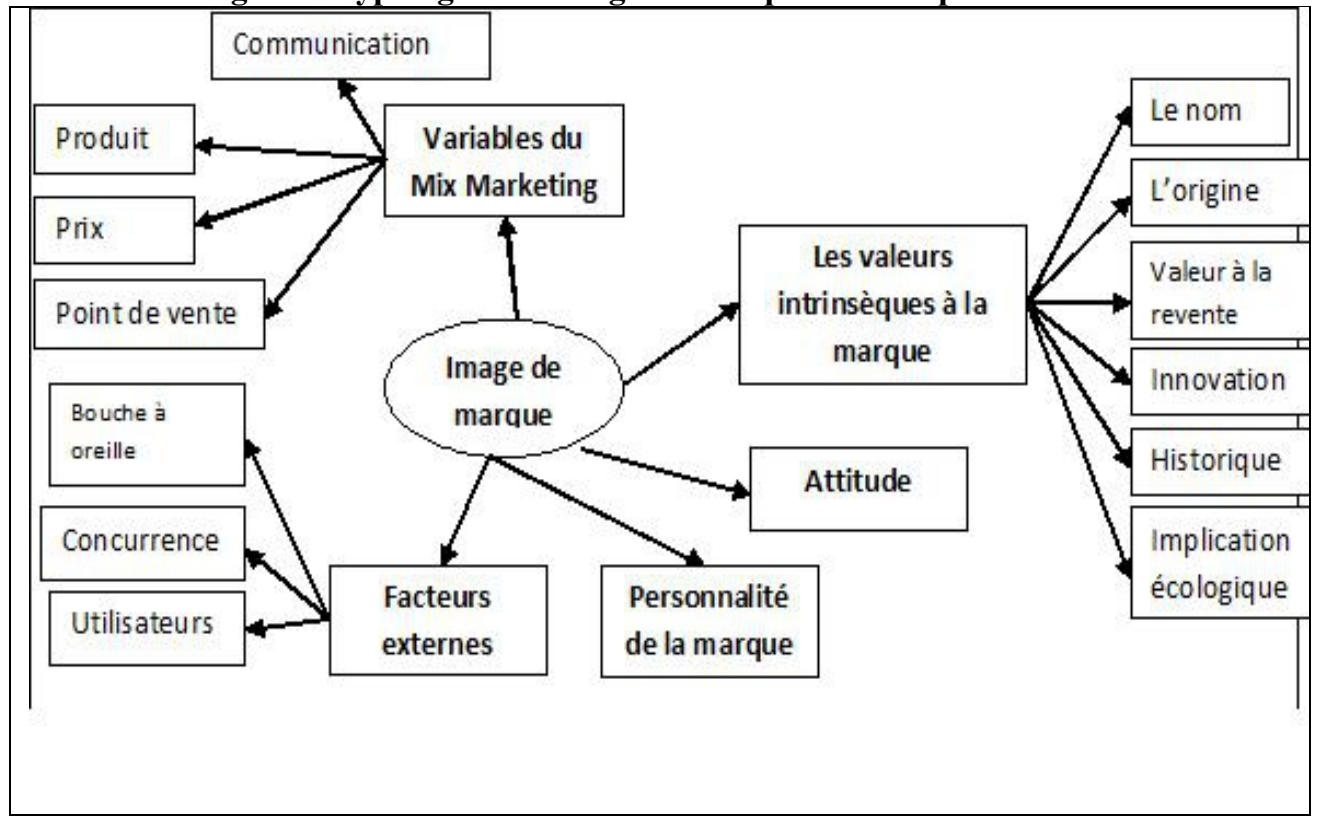

Conclusion

Source : conception personnelle

Ce papier résume les résultats de « l'étude exploratoire », qui a porté sur 23 interviewés. Celle-ci nous a permis d'examiner en détail les principales catégories relatives aux associations à la marque, dans le secteur automobile, au niveau du grand Agadir. Cela nous a amené à concevoir notre propre typologie de l'image de marque. En effet, la mesure de l'image de marque doit se faire en deux étapes, la première est dite exploratoire, moyennant une étude qualitative. Ce travail s'inscrit dans cette optique.

De plus, cette étude a été faite à l'instar de Keller, (1993), Aaker, (1994) et Korchia, (2001), qui ont développé des typologies de l'image de marque permettant sa mesure. Notre travail s'inscrit aussi dans la succession des études des auteurs cités, en élaborant notre propre typologie de l'image de marque, adaptée à notre contexte.

La deuxième étape consiste à mener une étude quantitative, dont les principaux axes doivent porter autour des composantes de la typologie réalisée. A cet effet, la mesure de l'image de marque automobile doit reposer sur les variables du mix marketing, les valeurs intrinsèques à la marques, les facteurs externes, la personnalité de la marque et l'attitude envers la marque.

Enfin, ce travail est limité à un contexte bien déterminé, de ce fait il serait très constructif de tester cette typologie sur un secteur différent. 


\section{References:}

1. Aaker, D.A. (1994). Le management du capital de marque, Dalloz.

2. Aaker, D.A. (2011). Building strong brands, the free press, New York.

3. Anderson, J. R. (1983). «A Spreading Activation Theory of Memory». Journal of Verbal Learning and Verbal Behavior.

4. Baumard, P. \& Ibert, J. (2007). Méthodes de recherche en management, 3ème édition, Dunod.

5. Brucks, M. (1985). «The Effects of Product Class Knowledge on Information Search Behavior», Journal of Consumer Research.

6. Demers, C. (2003). «L'entretien» Conduire un projet de recherche, une perspective qualitative, Collombelles : EMS.

7. Evrard, Y., Pras, B., \& Roux, E. (2000), Market, Dunod, Paris.

8. Heude, RP. (1990). L'image de marque, Ed. Eyrolles, Paris.

9. Keller, KL. (1993). «Conceptualizing, measuring, and managing customer-based brand equity» Journal of Marketing

10. Korchia, M. (2001). Connaissance des marques stockées en mémoire par les consommateurs: modèle théorique et test empirique, Thèse pour l'obtention du titre de docteur ès sciences de gestion, Marseille, IAE, Aix-Marseille III.

11. Loufrani-Fedida, S. (2006). Management des compétences et organisation par projets : une mise en valeur de leur articulation. Analyse qualitative de quatre cas multi sectoriels. Thèse de doctorat en Gestion et management. Université Nice Sophia Antipolis.

12. Michel, G. (2009). Au cœur de la marque, Collection Fonctions de l'entreprise, Dunod.

13. Miles, M. B. \& Huberman, M. A. (2003). Analyse des données qualitatives. (2e éd.). Paris : De Boeck.

14. Ratier, M. (2006). La perception de l'image d'une marque automobile par le consommateur : proposition d'un modèle, Thèse de doctorat en sciences de gestion, Toulouse 1 .

15. Thietard, R.A. (2014). Méthodes de recherche en management, Dunod. 